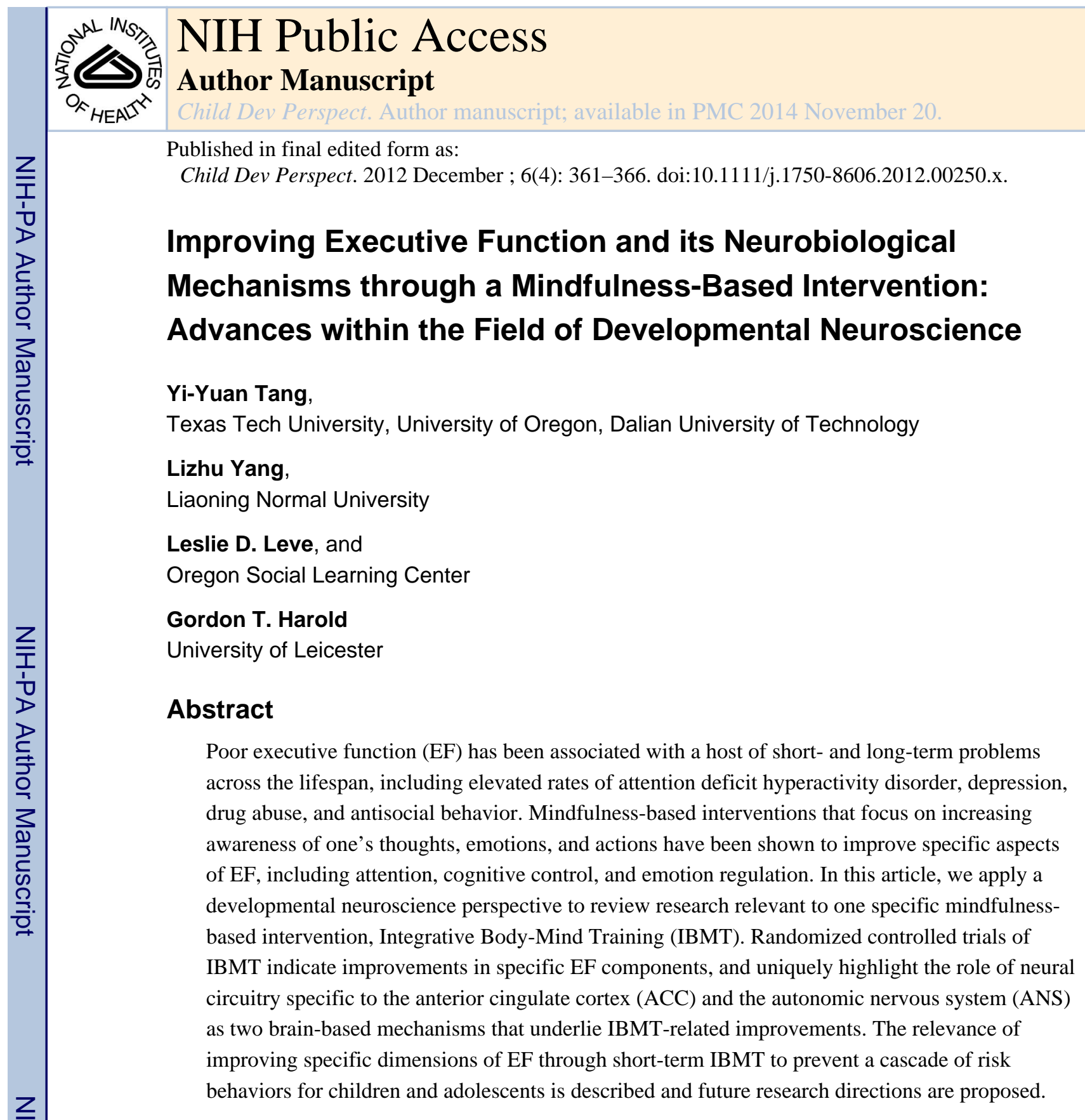

\title{
Keywords
}

mindfulness-based interventions; executive function; RCT; Integrative Body-Mind Training (IBMT); childhood; adolescent; adult

\footnotetext{
Executive function (EF) is a central component related to an individual's capacity to adaptively regulate his/her thoughts, emotions, instincts, and actions (Posner \& Rothbart, 2009). A key aspect of EF is the ability to mediate conflict between competing emotions or response tendencies. While $\mathrm{EF}$ also involves delay of reward, planning, implementation of coherent goals and many other processes, the ability to control competing tendencies is a central aspect of each of these EF process described above, and has been measured consistently both in cognitive studies (Botvinick, Braver, Barch, Carter, \& Cohen, 2001), and through the use of questionnaires that measure effortful control (Rothbart, 2011). As
} 
such, EF comprises a broad category of discrete but inter-related cognitive processes, including attentional control, attention shifting, cognitive flexibility, self-monitoring, planning, inhibitory control of prepotent responses, and working memory. In the current paper, we describe a mindfulness-based intervention focused on improving several specific components of $\mathrm{EF}$ (attentional control, self-regulation, ability to resolve conflicting information), behavioral correlates of these EF components (improved positive mood and reduced negative mood), and two neurobiological mechanisms that have been associated with these EF skills (the anterior cingulate cortex $[\mathrm{ACC}]$ and the autonomic nervous system [ANS]).

\section{Executive Function and Development}

Extant studies indicate robust associations between deficits in components of EF (i.e., selfregulation problems, attentional control difficulties) and a host of negative outcomes across the lifespan, including behavior problems, aggression, antisocial behavior, inattention, ADHD, problems with peers, school failure, depression, and substance abuse during childhood and adolescence (Eigsti et al., 2006; Floyd \& Kirby, 2001; Ivanov, Schulz, London, \& Newcorn, 2008; Mahone \& Hoffman, 2007; Moffitt et al., 2011; Perner, Kain, \& Barchfeld, 2002; Riggs, Blair, \& Greenberg, 2003). Conversely, higher levels of these EF components have been associated with positive developmental outcomes, including improved "on-task" behavior, better perspective taking skills, greater self-efficacy, mastery, and self-esteem (Blair \& Peters, 2003; Carlson, Mandell, \& Williams, 2004; Carlson \& Moses, 2001). Regulation and expression of emotionality appear to be critical underpinnings of adaptive EF development (Eisenberg et al, 1997; Gartstein \& Rothbart, 2003), and both $\mathrm{EF}$ and emotion-regulation skills show age-related changes across early childhood (Best, Miller, \& Jones, 2009; Lipscomb et al., 2011).

Adult outcomes of childhood EF have also been identified, with poor childhood EF (i.e., poor self-regulation) predicting poor economic outcomes, low job satisfaction, higher rates of relationship breakdown, and poorer physical health; conversely, high childhood EF predicts increased well being, professional attainment, and relationship success, as well as positive social, emotional, behavioral, economic, and physical health outcomes one to three decades later (Moffitt et al., 2011). These findings have brought increased attention to the development of intervention strategies aimed at improving EF proficiency early in development in order to promote more positive developmental outcomes later in life (Duckworth, 2011). In particular, there has been a surge in the development of mindfulnessbased interventions targeting improved developmental outcomes in children and adolescents (see Greenberg \& Harris, 2011, for a review). Below we describe one such intervention, Integrative Body-Mind Training (IBMT), and summarize evidence for its efficacy. Identifying mechanisms and interventions that promote the development of specific EF skills early in life may have substantial implications for the development of well-being across the life course. 


\section{Mindfulness-based Interventions Targeting EF and Associated Behaviors}

Mindfulness-based interventions (MBIs) are composed of several key components aimed at progressively orienting the participant to an awareness of the connection and synergy between body and mind. Techniques employed to attain this state include body relaxation, breathing practice, mental imagery, and body and mind awareness (Chiesa \& Malinowski, 2011; Kabat-Zinn, 1990; Lutz, Slagther, Dunne, \& Davidson, 2008; Tang, 2009; Tang et al., 2007, 2009; Tang \& Posner, 2009). Although MBIs vary in their methods, quality, and dosage, some have been shown to improve attention, emotion regulation, and social relationships in children and adults (Black, Milam, \& Sussman, 2009; Bowen et al., 2006; Chiesa \& Malinowski, 2011; Chiesa \& Serretti, 2010; Greenberg \& Harris, 2011; Lutz et al., 2008; Tang et al., 2007; Tang \& Posner, 2009; Zylowska et al., 2008). However, as noted in a recent review (Greenberg \& Harris, 2011), the majority of research with children and adolescents in this area has lacked scientific rigor. For example, a review by Birdee and colleagues (2009) identified 19 mindfulness-based interventions for children (yoga-based) and concluded that most did not adequately quantify key design elements, such as instructor qualifications, attrition, and randomization methods, and/or conduct appropriate data analysis. Below we summarize the scientific evidence for IBMT—one example of an MBI - across the lifespan. IBMT has been tested in several randomized clinical trials (RCTs) using relaxation training as a control (Tang, 2005, 2007, 2009; Tang et al., 2007, 2009, 2010). We begin by providing a brief description of the IBMT intervention; additional details of the intervention are available in Tang et al., (2007, 2009, 2010).

\section{The IBMT Intervention}

\section{IBMT core components}

The IBMT intervention involves body relaxation (e.g., conscious relaxation of muscle sets), mental imagery (e.g., imaging the calm sea), and mindfulness training (e.g., awareness of one's thought without judgment). The IBMT method stresses no effort to control one's thoughts, but rather a state of restful alertness is promoted that allows a high degree of awareness of one's body, breathing, mind, and external instructions. The goal of IBMT is to achieve a meditative state through the development of a strong mind-body connection; for example, relaxing the body and becoming increasingly aware of the relaxation feeling and sensations are two strategies that facilitate entry into the meditative state. The combined use of body and mind training is supported by studies of embodied cognition, in which changes in the body, particularly in facial expression, influence emotional processing, facilitate retrieval of autobiographical memories, and enhance feelings of personal self-efficacy and control (Dijkstra, Kaschak, Zwaan, 2007; Niedenthal, 2007; Huang, Galinsky, Gruenfeld, Guillory, 2011). A qualified IBMT coach who provides supporting instructions throughout the session guides participants in their practice. A qualified coach is an individual who has completed IBMT training and passed associated certification tests, has participated actively in the IBMT intervention as part of training, and has evidenced how to effectively interact with the trainees. The role and skill of the trainer in effectively engaging intervention participants (i.e., using a developmentally-appropriate approach to help participants enter a 
meditation state) is critical, especially when working with adolescents and children (Tang,

2005, 2007, 2009).

\section{IBMT procedures}

IBMT practice is typically conducted in a group setting. It includes (a) a pre-session (approximately $5 \mathrm{~min}$ ), (b) a practice session (approximately $20 \mathrm{~min}$ ), and (c) a post-session (approximately $5 \mathrm{~min}$ ). Each session is accompanied by a qualified IBMT coach. In the presession, the coach reviews the instructions for the practice session, interacts with the participants, and helps guide them into a quietness and mindfulness state. In the practice session, the participants follow IBMT instructions via a compact disk that includes background music to help the participants enter a meditative state. During the practice session, the coach observes facial and body cues to identify participants who are struggling with the method and provides feedback either promptly or after the session. The practice sessions are intended to help each participant increase the length of time spent in the meditation experience; thus, the quality of meditation generally improves with each session. In the post-session, the coach facilitates a brief group discussion to help ensure that participants have positive experiences consonant with the intent of IBMT (Tang, 2007, 2011; Tang et al, 2007, 2009).

Although the general IBMT procedures are similar across the lifespan, several modifications are made based on the age and/or ability of participants. For example, in older adults, the IBMT coach often instructs participants to practice each technique slowly. In adolescence, which represents an important transitional stage of emotional, behavioral, cognitive, and physical development that may be particularly responsive to mindfulness training, IBMT is modified such that the coach describes the scientific research results and explains the working mechanisms to help motivate the adolescents to follow the training. In young children, where evidence has shown that the preschool period is a critical time for the development of self-control, with long-term effects for children who show deficits in this area (e.g., Moffitt et al., 2011), IBMT is modified such that the coach uses cartoons or stories to create an environment to help the children enter the meditative state (Tang, 2007). In sum, the qualified coach plays a key role in facilitating different age groups of participants to enter and maintain in the meditation state (Tang, 2009; Tang et al., 2007).

\section{Specific EF-component and Behavioral Outcomes Associated with IBMT}

As noted above, several RCTs have been conducted to test the efficacy of IBMT. Below, we briefly review results of three studies at varying ages that compared IBMT and relaxation following periods of 5 to 30 days of training.

In Study 1, Chinese undergraduates were randomly assigned to either an experimental group (IBMT, $n=40$ ) or to an active control group $(n=40)$ using a pre- post-study design (Tang et al., 2007). Each participant was given 5 days of group training, consisting of $20 \mathrm{~min}$ per day. The training varied by condition: the experimental group was given 5 days of the IBMT intervention, and the control group was given the same amount of relaxation training. Relaxation training was guided by a tutor and compact disk and involved relaxing different muscle groups (e.g., face, head, shoulders, arms, legs, chest, back, abdomen, etc.). Analyses 
indicated that, after 5 days of training, the IBMT group showed significantly greater improvement in executive attention during the Attention Network Test (Fan, McCandliss, Sommer, Raz, \& Posner, 2002) than did the control group. In addition, individuals in the IBMT condition had lower negative affect and fatigue, and higher positive feelings on the Profile of Mood States (Shacham, 1983).

Study 2 extended the IBMT paradigm downward in development, and focused on middle and high school Chinese students. In this study, 489 students were given six weeks of 30min training per day of the IBMT intervention prior to their local and national entry examination. Compared to the randomized active control group, the IBMT practice improved attention (sustained attention and selective attention tasks), positive emotion, Raven's test and academic performance (scores of literacy, math and second language), as well as social behavior (Tang, 2005, 2009).

In Study 3, we tested the efficacy of IBMT in an even younger population: 60 preschoolaged children (ages 4-5 years) in China. We modified the IBMT intervention by using cartoons and stories to create an environment to help the children enter the meditative state. Results of this randomized controlled trial were consistent with the IBMT studies with adolescents and adults: 10 hrs of IBMT significantly increased children's self-control scores on the Child Behavior Questionnaire (Rothbart, Ahadi, Hershey, \& Fisher, 2001) and improved their executive function on two observed tasks in comparison with an active control condition that included a classroom activity (Diamond \& Lee, 2011). Overall, this programmatic set of three studies suggests that IBMT is effective across the lifespan in improving the specific components of EF.

\section{Neurobiological Mechanisms Underlying IBMT}

What are the mechanisms by which IBMT produces changes in specific EF components, including attention and self-regulation? As described above, IBMT is intended to modify brain states by increasing the brain-body connection of participants during the mediation session. Brain states refer to reliable patterns of brain activity that involve the activation or connectivity of multiple large-scale brain networks that are present even when doing no specific task (Bressler \& Menon, 2010). Brain state changes refer to the shift between certain forms of experience such as sleepiness or wakefulness, or meditation or exercise. These experiences share an altered state of mind and body. IBMT is hypothesized to improve attention and self-regulation through brain state changes involving both body and mind. Two indicators of brain state changes are increased activity in the prefrontal cortex of the brain, and changes in heart rate variability. Specifically, neuroimaging studies have indicated that discrete interrelated aspects of EF, including attentional control, attention shifting, cognitive flexibility, self-monitoring, planning, inhibitory control of prepotent responses, and working memory, are associated with activation of the prefrontal cortex (Roth, Randolph, Koven, \& Isquith, 2006; Wood \& Smith, 2008). In particular, the anterior cingulate cortex (ACC) is a key brain region in the prefrontal cortex that has been associated with EF (see reviews by Bush, Luu, \& Posner, 2000; Posner, Rothbart, Sheese, \& Tang, 2007). We therefore hypothesized that IBMT would show corresponding changes in the prefrontal cortex region of the brain (the ACC in particular), illustrating a neurobiological 
mechanism underlying the behavioral improvements in EF reviewed above. Second, changes in heart rate variability reflect activation of the autonomic nervous system (ANS), and we therefore hypothesized that IBMT would show effects on heart rate variability. Studies evidencing that these two neurobiological systems are activated by IBMT are summarized below.

In Study 4, we randomly assigned 46 Chinese undergraduates to IBMT or relaxation groups and conducted brain imaging assessments before, during, and after 5 days of IBMT and relaxation training (Tang et al., 2009). Neuroimaging data demonstrated that individuals in the IBMT condition showed stronger subgenual and adjacent ventral ACC activity compared to individuals in the control condition. In Study 5, a new sample of 40 Chinese undergraduates were randomly assigned to IBMT or relaxation groups, and EEG data and physiology data were collected before, during, and after 5 days of IBMT and relaxation training (Tang et al., 2009). Study 5 results indicated that frontal midline ACC theta correlated with high-frequency heart rate variability among individuals in the IBMT condition, suggesting control by the ACC over parasympathetic activity (Tang et al., 2009). Together, these studies indicate that both the ACC and ANS may serve as mediating brain mechanisms linking IBMT with improvements in EF.

We additionally collected physiological measures, including heart rate, skin conductance response, and respiratory rate and amplitude from Study 4 and Study 5 participants to monitor ANS activity (Tang et al., 2009). After 5 days of training, individuals in Study 4 and 5 in the IBMT group showed better ANS regulation by a ventral midfrontal brain system than did the relaxation group. This changed brain state may be a result of training in the coordination and balance of body and mind given in the IBMT but not in the control group (Tang et al., 2009). In addition, during and after training, the IBMT group across both studies showed significantly better physiological reactions in heart rate, respiratory amplitude and rate, and skin conductance response than the relaxation control group (Tang et al., 2009). Differences in heart rate variability and skin conductance response suggested greater involvement of ANS in the IBMT group during and after training.

Similar results were found in Study 3, where 20 of the 60 Chinese preschoolers originally participating in this study ( $n=10$ /group) were consented by parents to examine eventrelated potential (ERP) using a go/no-go task. Results indicated that the IBMT intervention significantly changed the amplitude of the ERP component P3 thought to involve the ACC (Ilan \&Polich, 1999). Children in the control condition did not show such effects, suggesting that the IBMT intervention produced inhibitory control improvements associated with better executive function and self-regulation (Yang, Song, Shen, Cui, \& Tang, 2010).

\section{Dosage effects}

As noted in recent reviews (e.g., Greenberg \& Harris, 2011), many existing mindfulnessbased interventions fail to consider dosage effects. To address this issue, we conducted a sixth study that comprised 4 weeks of training (11 hrs total) using a randomized controlled trial design to compare IBMT to a relaxation training control condition. As described above, IBMT studies 1-5 indicated that, with less than $3 \mathrm{hrs}$ of total training in IBMT, individuals saw improvements in attention and self-control, increases in positive emotion, reductions in 
stress and cortisol reactivity, and improved immune function through a central (brain) and autonomic (body) nervous system function (Tang et al., 2007, 2009). Study 6 addressed the questions of whether there are additional potential benefits of IBMT practice when implemented over a longer period of time. In Study 6, compared to relaxation training, 4 weeks of IBMT significantly improved the efficiency of alerting and executive attention networks (Tang, 2009). In contrast, the 1-week IBMT only improved the executive attention network (Tang et al., 2007). On the basis of this evidence, we next tested the hypothesis that $11 \mathrm{hrs}$ of training with IBMT over 4 weeks would increase fractional anisotropy, an index representing the integrity and efficiency of white matter in the corona radiata, an important white-matter tract connecting the ACC to other structures. In Study 7, we randomized 45 U.S. undergraduates to an IBMT or relaxation group and acquired brain images from each participant at rest using MRI diffusion tensor imaging for analysis of white matter before and after training. Results showed that $11 \mathrm{hrs}$ of IBMT increased fractional anisotropy connecting ACC to other regions. Because deficits in activation of the ACC have been associated with many disorders including mood disorders and substance abuse, the ability to strengthen cingulate connectivity through training could provide a means for improving EF and might serve as a possible therapy or prevention tool (Tang et al., 2010).

\section{Summary and Future Directions}

Deficiencies in EF are associated with a host of problems across the lifespan, such as mood disorders, school failure, substance abuse, and antisocial behavior. The series of RCT studies of IBMT described in this paper indicate the positive effects of IBMT on several components of $\mathrm{EF}$, including attentional control, emotion regulation, and response to stress (Tang, 2005, 2007, 2009, 2011; Tang et al., 2007, 2009, 2010; Tang \& Posner, 2009). In addition, this work highlights the ACC and the ANS as neurobiological mechanisms underlying the behavioral changes seen in specific EF components through IBMT. Recent studies also indicate the important role of ACC in MBIs (for a review, see Hölzel et al., 2011). An advantage of IBMT is that it has been tested with randomized trials that have included an active control condition consisting of relaxation training, which is often used to improve performance. As such, the effects of the IBMT intervention cannot simply be due to contact with a trained interventionist, but rather, are specific to the components of the IBMT intervention. As reviewed above, changes induced by IBMT can occur after as little as 5 days of training, but are greater with longer training periods. However, it is unknown the extent to which the effects of IBMT persist over time, a year or more after training. This research has implications for the prevention of risky behaviors such as drug use; for example, if we can implement IBMT or other effective MBIs in at-risk populations of children, we can improve specific components of EF, including self-regulation and attentional control, that may then prevent the development of subsequent problems such as school failure and substance use.

IBMT studies conducted to date have included normative or community-based samples rather than samples indicated for clinical problems such as ADHD. The theory underlying IBMT would suggest similar improvements in EF in clinical populations, but this important work has yet to be conducted. We do not know which features of IBMT are of greatest importance in obtaining change. We believe that the integration of the various mindfulness 
components into one single training package may explain why IBMT is effective at such a low dose, but the components have yet to be tested individually. The role of the IBMT trainer could also be a central effective ingredient, and thus requires additional research (Tang, 2009; Tang et al., 2007, 2010; Posner, Rothbart, Rueda, \& Tang, 2010).

In summary, IBMT is one of many MBIs. Although its benefits have been verified using random assignment trials that have included a relaxation control condition, additional research and intervention development is needed with not only IBMT, but also with other promising MBIs, given the preliminary evidence for such interventions to improve EF and associated behavioral outcomes.

\section{Acknowledgments}

We thank the staff at the Institute of Neuroinformatics for assistance in data collection, and Michael Posner for insightful comment and revision. This work and the writing of this report were supported by 973 Program 2012CB518200, Office of Naval Research, R21 DA030066, P30 DA023920, and R01 HD042608.

\section{References}

Birdee GS, Yeh GY, Wayne PM, Phillips RS, David RB, Gardiner P. Clinical applications of yoga for the pediatric population: A systematic review. Academic Pediatrics. 2009; 9:212-220. [PubMed: 19608122]

Best JR, Miller PH, Jones LL. Executive functions after age 5: Changes and correlates. Developmental Review. 2009; 29:180-200. [PubMed: 20161467]

Black DS, Milam J, Sussman S. Sitting-meditation interventions among youth: A review of treatment efficacy. Pediatrics. 2009; 124:532-541.

Blair C, Peters R. Physiological and neurocognitive correlates of adaptive behavior in preschool among children in Head Start. Developmental Neuropsychology. 2003; 24:479-497. [PubMed: 12850755]

Botvinick MM, Braver TS, Barch DM, Carter CS, Cohen JD. Conflict monitoring and cognitive control. Psychological Review. 2001; 108:624-652. [PubMed: 11488380]

Bowen S, Witkiewitz K, Dillworth TM, Chawla N, Simpson TL, Ostafin BD, Blume AW. Mindfulness meditation and substance use in an incarcerated population. Psychology of Addictive Behaviors. 2006; 20:343-347. [PubMed: 16938074]

Bressler SL, Menon V. Large-scale brain networks in cognition: emerging methods and principles. Trends in Cognitive Sciences. 2010; 14:277-290. [PubMed: 20493761]

Bush G, Luu P, Posner MI. Cognitive and emotional influences in anterior cingulate cortex. Trends in Cognitive Sciences. 2000; 4:215-222. [PubMed: 10827444]

Carlson SM, Mandell DJ, Williams L. Executive functioning and theory of mind: Stability and prediction from ages 2 to 3. Developmental Psychology. 2004; 40:1105-1122. [PubMed: 15535760]

Carlson SM, Moses LJ. Individual differences in inhibitory control and children's theory of mind. Child Development. 2001; 72:1032-1053. [PubMed: 11480933]

Chiesa A, Malinowski P. Mindfulness-based approaches: are they all the same? Journal of Clinical Psychology. 2011; 67:404-424. [PubMed: 21254062]

Chiesa A, Serretti A. A systematic review of neurobiological and clinical features of mindfulness meditations. Psychological Medicine. 2010; 40:1239-1252. [PubMed: 19941676]

Diamond A, Lee K. Interventions shown to aid executive function development in children 4 to 12 years old. Science. 2011; 333:959-964. [PubMed: 21852486]

Dijkstra K, Kaschak MP, Zwaan RA. Body posture facilitates retrieval of autobiographical memories. Cognition. 2007; 102:139-149. [PubMed: 16472550]

Duckworth AL. The significance of self-control. Proceedings of the National Academy of Sciences. 2011; 108:2639-2640. 
Eigsti I, Zayas V, Mischel W, Shoda Y, Ayduk O, Dadlani MB, Casey BJ. Predictive cognitive control from preschool to late adolescence and young adulthood. Psychological Science. 2006; 17:478484. [PubMed: 16771797]

Eisenberg N, Guthrie IK, Fabes RA, Reiser M, Murphy BC, Holgren R, Losoya S. The relations of regulation and emotionality to resiliency and competent social functioning in elementary school children. Child Development. 1997; 68:295-311. [PubMed: 9180003]

Fan J, McCandliss BD, Sommer T, Raz A, Posner MI. Testing the efficiency and independence of attentional networks. Journal of Cognitive Neuroscience. 2002; 14:340-347. [PubMed: 11970796]

Fan Y, Tang YY, Ma Y, Posner MI. Mucosal immunity modulated by integrative meditation in a dose dependent fashion. Journal of Alternative and Complementary Medicine. 2010; 16:151-155.

Floyd RG, Kirby EA. Psychometric properties of measures of behavioral inhibition with preschool-age children: Implications for assessment of children at risk for ADHD. Journal of Attention Disorders. 2001; 5:79-91.

Garstein MA, Rothbart MK. Studying infant temperament via the revised infant behavior questionnaire. Infant Behavior \& Development. 2003; 26:64-86.

Greenberg MT, Harris AR. Nurturing mindfulness in children and youth: Current state of research. Child Development Perspectives. 2011 Online first.

Hölzel BK, Lazar SW, Gard T, Schuman-Olivier Z, Vago DR, Ott U. How does mindfulness meditation work? Proposing mechanisms of action from a conceptual and neural perspective. Perspectives on Psychological Science. 2011; 6:537-559.

Huang L, Galinsky AD, Gruenfeld DH, Guillory LE. Powerful postures vs. powerful roles: Which is the proximate correlate of thought and behavior? Psychological Science. 2011; 222:95-102. [PubMed: 21149853]

Ilan AB, Polich J. P300 and response time from a manual Stroop task. Clinical Neurophysiology. 1999; 110:367-373. [PubMed: 10210626]

Ivanov I, Schulz KP, London ED, Newcorn JH. Inhibitory control deficits in childhood and risk for substance use disorders: A review. American Journal of Drug and Alcohol Abuse. 2008; 34:239258. [PubMed: 18428067]

Kabat-Zinn, J. Full catastrophe living: Using the wisdom of your body and mind to face stress, pain and illness. New York: Delacorte; 1990.

Lipscomb ST, Leve LD, Harold GT, Neiderhiser JM, Shaw DS, Ge X, Reiss D. Trajectories of parenting and child negative emotionality during infancy and toddlerhood: A longitudinal analysis. Child Development. 2011; 82:1661-1675. [PubMed: 21883160]

Lutz A, Slagter HA, Dunne JD, Davidson RJ. Attention regulation and monitoring in meditation. Trends in Cognitive Science. 2008; 12:163-169.

Mahone EM, Hoffman J. Behavior ratings of executive function among preschoolers with ADHD. Clinical Neuropsychology. 2007; 21:569-586.

Moffitt TE, Arseneault L, Belsky D, Dickson N, Hancox RJ, Harrington H, Caspi A. A gradient of childhood self-control predicts health, wealth, and public safety. Proceedings of the National Academy of Sciences. 2011; 108:2693-2698.

Niedenthal PM. Embodying emotion. Science. 2007; 316:1002-1005. [PubMed: 17510358]

Perner J, Kain W, Barchfeld P. Executive control and higher-order theory of mind in children at risk of ADHD. Infant and Child Development. 2002; 11:141-158.

Posner MI, Rothbart MK. Toward a physical basis of attention and self regulation. Physical Life Review. 2009; 6:103-120.

Posner, MI.; Rothbart, M.; Rueda, MR.; Tang, YY. Training effortless attention. In: Bruya, B., editor. Effortless Attention: a new perspective in the cognitive science of attention and action. Cambridge, MA: MIT Press; 2010.

Posner MI, Rothbart MK, Sheese BE, Tang YY. The anterior cingulate gyrus and the mechanism of self-regulation. Cognitive, Affective \& Behavioral Neuroscience. 2007; 7:391-395.

Riggs NR, Blair CB, Greenberg MT. Concurrent and 2-year longitudinal relations between executive function and the behavior of $1^{\text {st }}$ and $2^{\text {nd }}$ grade children. Child Neuropsychology. 2003; 9:267-276. [PubMed: 14972705] 
Roth, RM.; Randolph, JJ.; Koven, NS.; Isquith, PK. Neural substrates of executive functions: Insights from functional neuroimaging. In: Dupri, JR., editor. Focus on neuropsychology research. Hauppauge, NY: Nova Science Publishers; 2006. p. 1-36.

Rothbart, MK. Becoming who we are: Temperament and personality in development. New York, NY: Guilford Press; 2011.

Rothbart MK, Ahadi SA, Hershey K, Fisher PA. Investigations of temperament at three to seven years: The Children's Behavior Questionnaire. Child Development. 2001; 72:1394-1408. [PubMed: 11699677]

Shacham S. A shortened version of the Profile of Mood States. Journal of Personality Assessment. 1983; 47:305-306. [PubMed: 6886962]

Tang, YY. Health from Brain, Wisdom from Brain. Dalian University of Technology Press (including training and practice VCDs) (in Chinese); 2005.

Tang, YY. Multi-intelligence and Unfolding the Full Potentials of Brain (including training and practice CD). Dalian University of Technology Press (in Chinese); 2007.

Tang, YY. Exploring the brain, Optimizing the life. Beijing, China: Science Press (in Chinese); 2009.

Tang YY. Mechanism of Integrative Body-Mind Training. Neuroscience Bulletin. 2011; 27:383-388. [PubMed: 22108815]

Tang YY, Lu Q, Geng X, Stein EA, Yang Y, Posner MI. Short-term meditation induces white matter changes in the anterior cingulate. Proceedings of the National Academy of Sciences. 2010; 107:15649-15652.

Tang YY, Ma Y, Fan Y, Feng H, Wang J, Feng S, Fan M. Central and autonomic nervous system interaction is altered by short term meditation. Proceedings of the National Academy of Sciences. 2009; 106:8865-8870.

Tang YY, Ma Y, Wang J, Fan Y, Feng S, Lu Q, Posner MI. Short-term meditation training improves attention and self-regulation. Proceedings of the National Academy of Sciences. 2007; 104:1715217156.

Tang YY, Posner MI. Attention training and attention state training. Trends in Cognitive Sciences. 2009; 13:222-227. [PubMed: 19375975]

Wood, AG.; Smith, E. Pediatric neuroimaging studies: A window to neurocognitive development of the frontal lobes. In: Anderson, V.; Jacobs, R.; Anderson, PJ., editors. Executive functions and the frontal lobes: A lifespan perspective. Philadelphia, PA: Taylor \& Francis; 2008. p. 203-216.

Yang, L.; Song, F.; Shen, Y.; Cui, Y.; Tang, YY. Integrative body-mind training improves selfregulation in children; Paper presented at the 7th International Conference on Cognitive science; Beijing, China. 2010 Aug.

Zylowska L, Ackerman DL, Yang MH, Futrell JL, Horton NL, Hale TS, Smalley SL. Mindfulness meditation training in adults and adolescents with ADHD: A feasibility study. Journal of Attention Disorders. 2008; 11:737-746. [PubMed: 18025249] 